\title{
Tecnura
}

\section{Sistema de gestión de inventario de sustancias agotadoras de la capa de ozono}

\section{Ozone depleting substances management inventory system}

\author{
Yelena Islen San Juan, Armando Gómez Díaz², Felix Ivan Romero Rodríguez³
}

Fecha de recepción: 26 de marzo de 2017

Fecha de aceptación: 28 de agosto de 2017

Cómo citar: San Juan, Y.I., Gómez D., A.L. y Romero R., F.I. (2017). Sistema de gestión de inventario de sustancias agotadoras de la capa de ozono. Tecnura, 21(54), 59-67. https://doi.org/10.14483/22487638.12258

\section{Resumen}

Contexto: El cuidado de la capa de ozono contribuye a la estabilidad ambiental del planeta. Por ello, desde el punto de vista organizativo, se crea el protocolo de Montreal, destinado a controlar la emisión de las sustancias agotadoras de la capa de ozono y reducir su producción. Sin embargo, es necesario tener el control de las que ya están en circulación y presentes en aquellos equipos que aún no son sustituibles debido al contexto de las empresas que los mantienen. Generalmente, los mecanismos de control para el clasificado del tipo de sustancias, los equipos y las empresas que los poseen, son llevados en archivos físicos, hojas de cálculo y documentos de textos, lo que dificulta el control y la gestión de los datos archivados en ellos. La presente investigación tiene como objetivo realizar la informatización del proceso de control de las sustancias agotadoras de la capa de ozono.

Método: Se realiza una evaluación y descripción de los procesos involucrados en la gestión de las sustancias agotadoras de la capa de ozono y sus alternativas. Para la informatización se utiliza la metodología de desarrollo ágil SCRUM y para la solución tecnológica, herramientas y tecnologías libres de código abierto.
Resultados: Como resultado de la investigación, fue desarrollada una herramienta informática que automatiza el proceso de control y gestión de las sustancias agotadoras de la capa de ozono y sus alternativas.

Conclusiones: La herramienta informática desarrollada permite controlar y gestionar las sustancias agotadoras de la capa de ozono y los equipos que las utilicen. De igual manera gestiona las sustancias que surgen como alternativas a utilizarse en aras de la protección de la capa de ozono.

Palabras clave: Informática, Ozono, Sustancias agotadoras de la capa de ozono.

\section{abstract}

Context: The care of the ozone layer is an activity that contributes to the planet's environmental stability. For this reason, the Montreal Protocol is created to control the emission of substances that deplete the ozone layer and reduce its production from an organizational point of view. However, it is also necessary to have control of those that are already circulating and those present in the equipment that cannot be replaced yet because of the context of the companies that keep it. Generally, the control mechanisms for classifying the type of substances, equipment and companies that

1 Ingeniera en Ciencias Informáticas, estudiante de doctorado, especialista B en Ciencias Informáticas, CITMATEL. La Habana, Cuba. Contacto: yelena870513@gmail.com

2 Ingeniero Mecánico, OTOZ. La Habana, Cuba. Contacto: mandy@cubaenergia.cu

3 Ingeniero en Ciencias Informáticas, estudiante de doctorado, especialista A en Ciencias Informáticas. Zunzun Education Ltd. La Habana, Cuba. Contacto: firomerorom4@gmail.com 
own them, are carried in physical files, spreadsheets and text documents, which makes it difficult to control and manage the data stored in them.

Method: The objective of this research is to computerize the process of control of substances that deplete the ozone layer. An evaluation and description of all process to manage Ozone-Depleting Substances (ODS), and its alternatives, is done. For computerization, the agile development methodology SCRUM is used, and for the technological solution tools and free open source technologies are used.
Result: As a result of the research, a computer tool was developed that automates the process of control and management of substances that exhaust the ozone layer and its alternatives.

Conclusions: The developed computer tool allows to control and manage the ozone-depleting substances and the equipment that use them. It also manages the substances that arise as alternatives to be used for the protection of the ozone layer.

Keywords: Information technology, Ozone, Ozone depleting substances.

\section{INTRODUCCIÓN}

La preservación de la capa de ozono es una tarea a cumplir por cada empresa que en su gestión diaria posee equipos o maneja sustancias que sensibilizan su estado. Con ese objetivo se crea el protocolo de Montreal (PNUMA, 2007), a fin de establecer un mecanismo que reduzca las emisiones de dichas sustancias y su consecuente control. Dichas sustancias fueron clasificadas y dadas a conocer (Montzka et al., 2011) para la gestión y control. La Unidad de Servicios de Investigación (USR) propone un mecanismo para la gestión de las sustancias y su clasificación (URS, 2009), el cual sugiere varias actividades que en su conjunto lideran a la organización del control de las Sustancias Agotadoras de la capa de Ozono (SAO). La USR en su mecanismo divide las actividades en varios procesos:

- Ámbito regulatorio: Cubre el servicio, mantenimiento de los equipos que contienen SAO, así como las restricciones de venta. Tiene la aplicación de estos a nivel industrial o personal.

- Procedimientos: Regula el manejo de equipos, así como establece los procedimientos para ello. De igual manera se tiene en cuenta la capacitación del personal y el trabajo por procedimientos certificados.
- Condiciones de reparación: Establece las condiciones de reparación de equipos que contengan SAO.

- Reportes: Establece los reportes a realizar sobre adquisición y control de las SAO. Mantiene el cálculo de fugas y fechado de cada operación.

En el caso de la gestión de sustancias químicas, de manera general, se tienen procedimientos y clasificaciones según su naturaleza (Miller et al., 2004). De esta manera se mantiene separado por áreas, nivel de afectación, lo que permite optimizar su utilización en dependencia de su contexto.

Una vez realizados estos procesos, se organiza el control de las sustancias; sin embargo, la centralización de la información puede tener errores de los datos cuando se realiza de manera manual. De igual forma, el mantenimiento (consulta, adición, edición y eliminación) de la información en hojas de cálculo se vuelve engorroso con el crecimiento de los datos. Asimismo, unificar la información de varias entidades que manejen información sobre equipos y sustancias SAO, se complejiza y ralentiza si se realiza manualmente, aumentando el riesgo de introducir errores. A partir de la problemática planteada, la presente investigación tiene como objetivo informatizar el proceso de levantamiento de inventario de las sustancias SAO, HFC y alternativas. 


\section{METODOLOGÍA}

La identificación de las sustancias SAO que generalmente se utilizan y su clasificación constituye el primer paso para su gestión (Intergovernmental Panel on Climate Change, y Grupo de Evaluación Tecnológica y Económica, 2005). Una vez realizado el mecanismo de clasificación, se hace necesario tener en cuenta cómo gestionar y controlar cada activo involucrado (URS, 2009) desde la empresa, equipos, servicios y personal. A continuación, se definen y refinan los procesos, de manera que no quede información sin captar (tabla 1). Una vez establecida la clasificación de la información (Intergovernmental Panel on Climate Change, y Grupo de Evaluación Tecnológica y Económica, 2005), se modela conceptualmente cómo relacionar todos los datos disponibles.
Una vez determinados los conceptos, se modela el dominio del uso y control de las SAO y los equipamientos que la tienen, a fin de componer los procesos que se involucran a diario.

La figura 1 muestra cómo se realiza este proceso, donde en la empresa cada especialista mantiene un inventario de equipos de aire acondicionado y de refrigeración. A su vez, lleva el control de las sustancias SAO, HFC y sus alternativas que contienen dichos equipos. Luego se colecta toda la información por empresa, generando información más generalizada que muestra una visión global de la utilización de las SAO por regiones y a nivel nacional, respecto empresas, organismos y ministerios involucrados. Teniendo en cuenta cuáles son las actividades y cómo se complementan, así como los datos que se necesitan, se lleva a cabo el proceso de informatización.

Tabla 1. Equipos con sustancias SAO

\begin{tabular}{ll}
\hline \multicolumn{1}{c}{ Equipos de aire acondicionado } & \multicolumn{1}{c}{ Equipos de refrigeración } \\
\hline Aire acondicionado de ventana & Vehículos de carretera refrigerados para transporte refrigerado \\
\hline Aire acondicionado en autos y pequeños vans & Los contenedores refrigerados en el transporte (estáticos) \\
\hline Aire acondicionado en camiones (referencia a cabinas) & Los equipos autónomos de refrigeración comercial \\
\hline Aire acondicionado en grandes vehículos & Los grandes sistemas de refrigeración industrial \\
\hline Aire acondicionado en tractores (referencia a cabinas) & Refrigeradores domésticos \\
\hline Pequeño autónomo (portátil) & Refrigeradores domésticos y freezers \\
\hline Pequeño autónomo (deshumidificadores) & Refrigeradores freezer \\
\hline Mini Split (casete) & Sistemas de Chiller industrial \\
\hline Mini Split (ocultos) & Sistemas de refrigeración por barcos \\
\hline Mini Split (otros) & \\
\hline Split & \\
\hline Sistemas de flujo de refrigerante variable & \\
\hline Consolas & \\
\hline
\end{tabular}

Fuente: elaboración propia. 


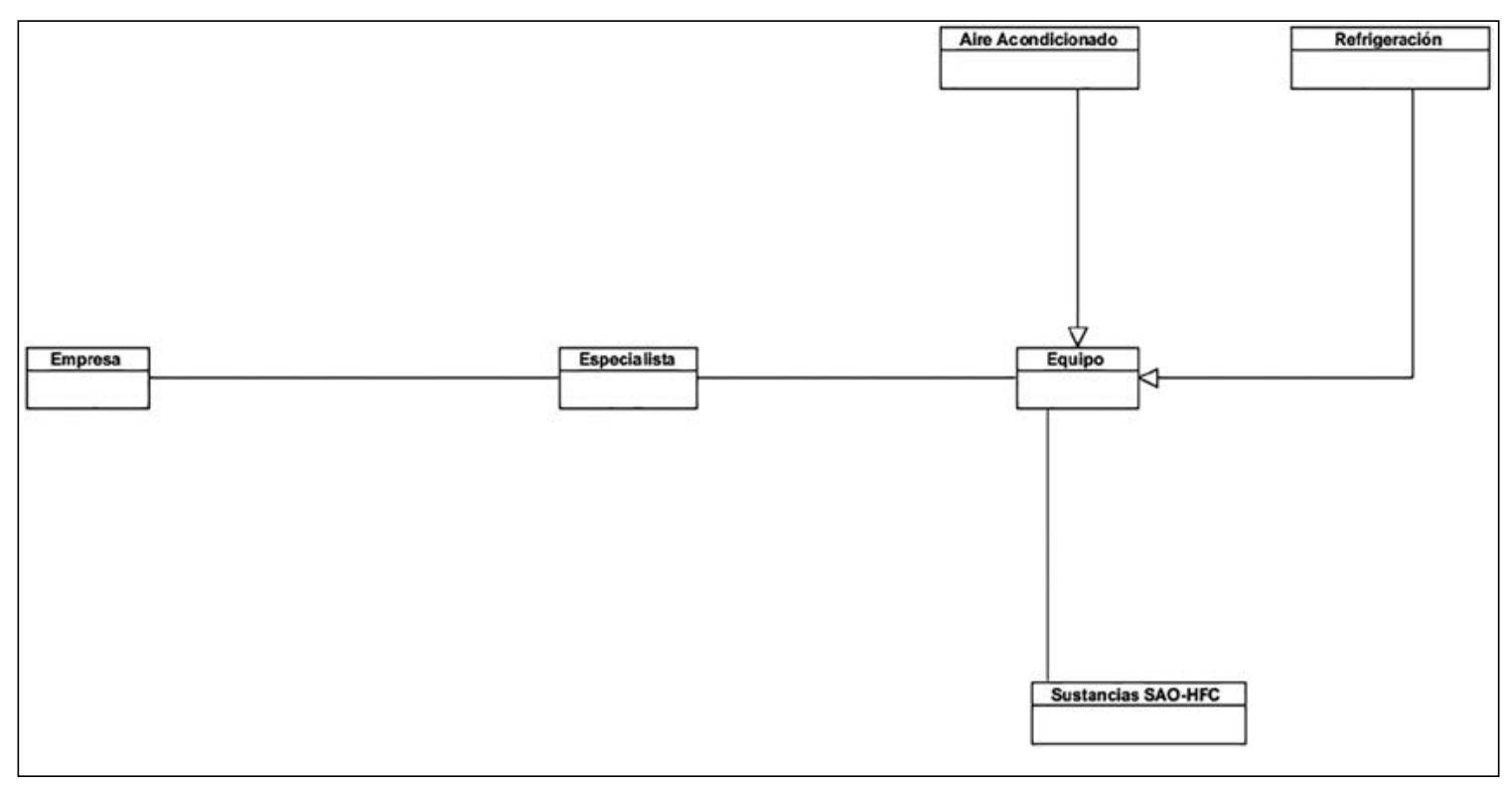

Figura 1. Funcionamiento del proceso de control de SAO

Fuente: elaboración propia.

\section{Herramientas}

\section{Metodología de desarrollo}

En el desarrollo, la metodología de software seleccionada tiene un impacto fundamental en la concepción de un proyecto informático. En el caso de Scrum (Schwaber y Sutherland, 2013), es un marco de trabajo, donde no necesariamente se cuenta con un plan detallado del producto, el equipo de desarrollo es autoorganizado y con un mecanismo de autocontrol en aras de mantener la creatividad de los integrantes del equipo. Scrum fue la metodología seleccionada para la herramienta en el control de las SAO.

\section{Sistema para la gestión de bases de datos}

La persistencia de los datos constituye otros de los parámetros que influyen en el desarrollo de sistemas. Generalmente el manejo de los datos se hace a través de los sistemas gestores de base de datos. En el desarrollo software existen tendencias para el almacenamiento de los datos. En los sistemas de gestión se utilizan las bases de datos relacionales y las no relacionales, comúnmente conocidas como bases de datos documentales (Pokorny, 2013). En el contexto del desarrollo de esta herramienta se pretende como particularidad del gestor de base de datos, sincronizar los registros existentes y el almacenamiento en forma de documentos en aras de facilitar la recuperación de datos. Teniendo en cuenta dichos requisitos, se selecciona para la construcción de la aplicación PouchDB (Smith y Harvey, 2017). Base de datos documental que permite la indización de sus registros y la creación de diseño de documentos para la recuperación de datos.

\section{Tecnologías de desarrollo}

La selección de las tecnologías de desarrollo es siempre un tema de debate entre los equipos de desarrollo (Rivas et al., 2010). Encontrar la tecnología adecuada pone en la mesa de trabajo diversos criterios a tener cuenta, como:

- Facilidad de implantación.

- Curva de aprendizaje.

- Costo de desarrollo.

- Soporte y transferencia. 
Teniendo en cuenta dichos criterios, para su desarrollo se selecciona como plataforma de desarrollo Electron (GitHub, 2016). Esta genera aplicaciones de escritorio utilizando tecnología web para ello. A partir de ahí, se selecciona como lenguaje de programación Node.js (Xalambrí, 2016) y AngularJS (Freeman, 2014).

Una vez seleccionada la tecnología es importante tener en cuenta las buenas prácticas (Romero y San Juan, 2017), y el uso correcto de patrones arquitectónicos y de diseño, así como mecanismos para el trabajo con los datos. El seguimiento se hace con el sistema de control de versiones Git (Chacon, 2014), para llevar el histórico del proyecto.

La base del sistema se desarrolla como una aplicación web, implementando toda transferencia de datos a través del protocolo HTTP (Gómez,
Ordóñez y Solarte, 2015). Como lenguaje de programación se utilizó JavaScript, sobre el cual están sustentadas la mayoría de las tecnologías utilizadas. El intercambio de datos se realiza mediante Ajax (Poveda, González, Velandia y Villalobos, 2013), con el componente HTTP que brinda el marco de trabajo AngularjS.

\section{Resultados}

Para lograr un desarrollo rápido se precisa de metodologías o procesos que amortigüen los problemas que han existido durante el desarrollo de software (Díaz et al., 2014). Para el levantamiento de las SAO, HFC y alternativas se emplea la metodología de desarrollo ágil SCRUM, donde se definen las principales fases, reuniones y revisiones con las herramientas seleccionadas para ello (figura 2).

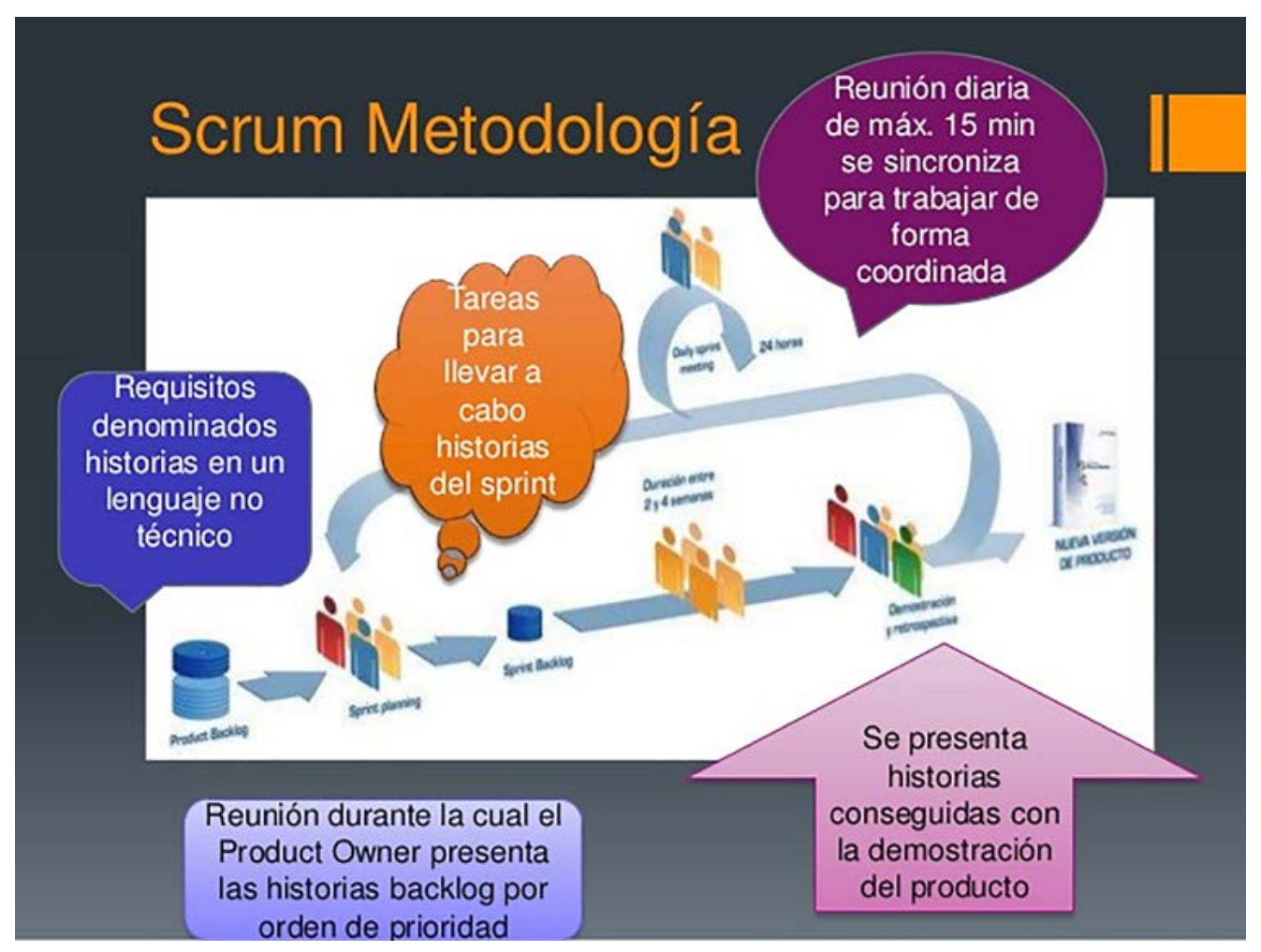

Figura 2. Metodología de desarrollo ágil SCRUM

Fuente: Palacios (2012). 
El software quedó conformado por los siguientes módulos:

- Usuario: Gestiona la seguridad de los usuarios del sistema.

- Nomencladores: Gestiona los nomencladores del sistema según su tipo.

- Gráficas: Realiza un reporte a través de gráficas, según los distintos aspectos a controlar de las SAO.

- Reportes: Realiza tablas de reportes según los indicadores de las SAO y los equipamientos. Permite exportar o imprimir los reportes generados.

- Operaciones: Realiza las operaciones para la centralización de los datos, como exportar, importar y limpiar base de datos.

- Gestión de las SAO: Mediante este se realiza la gestión integral de las SAO. Las operaciones que se hacen dentro de este módulo son:
- Inventario de equipo de clima y refrigeración.

- Inventario empresa de servicios del clima y refrigeración.

- Recolección de datos sobre el uso de alternativas de SAO en el sector de solventes.

- Demanda de SAO y refrigerantes alternativos de SAO.

- Consumo de SAO y sus alternativas en la fabricación de aire acondicionado.

- Consumo de SAO y sus alternativas en la fabricación de refrigerante.

- Consumo de SAO y sus alternativas en la fabricación de aerosoles.

El sistema generado para el levantamiento de las SAO, HFC y alternativas (San Juan, 2017) ha sido desarrollado completamente sobre tecnologías libres (figura 3, figura 4 y figura 5).

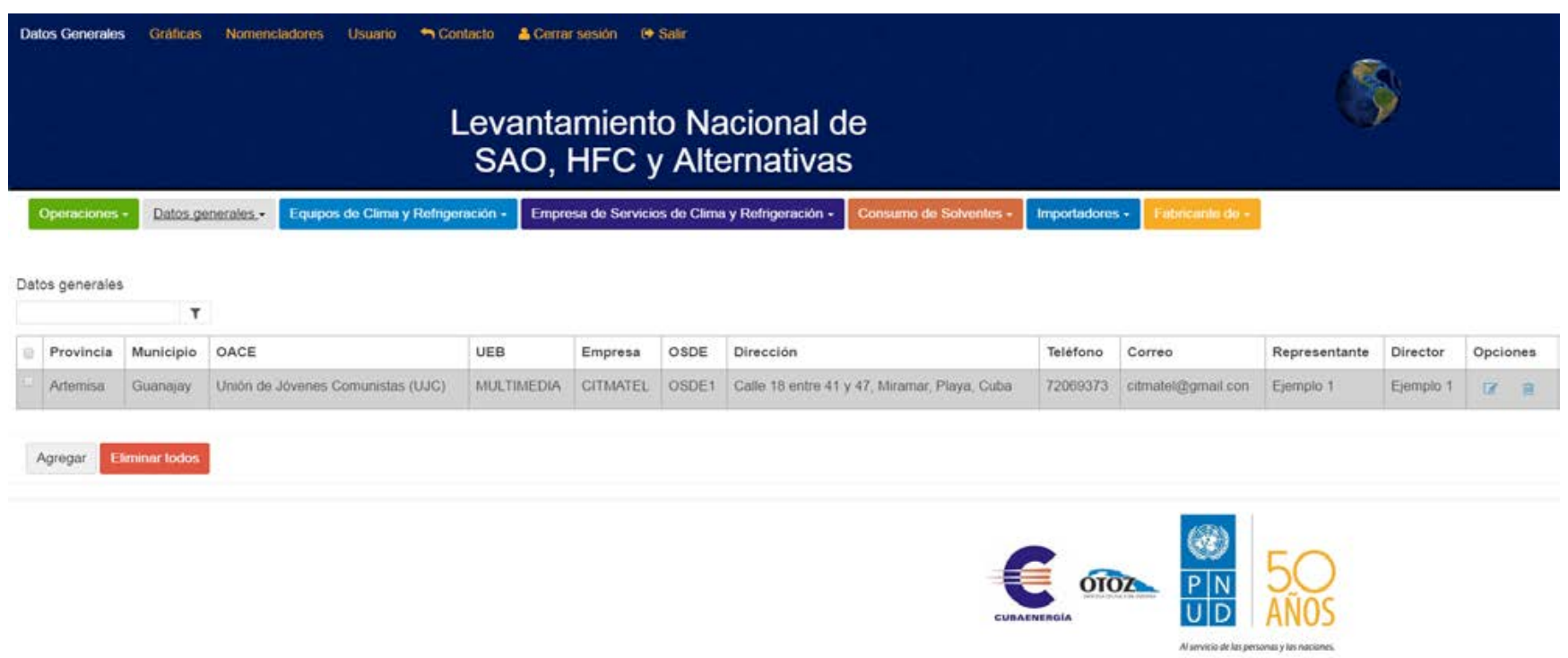

Figura 3. Vista de gestión de la herramienta desarrollada

Fuente: elaboración propia. 


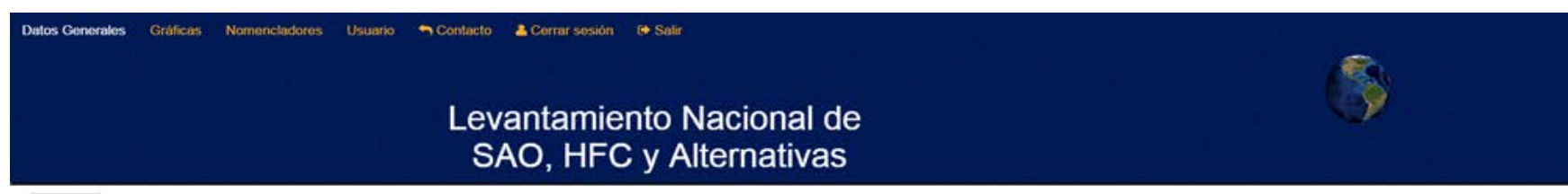

\begin{tabular}{|c|c|c|c|c|c|c|c|c|c|c|}
\hline Provincia & Municipio & OACE & UEB & Empresa & OSDE & Dirección & Telefono & Correo & Representante & Director \\
\hline Artemisa & Guanajay & $\begin{array}{l}\text { Ministerio del Trabajo y Sogundad Social } \\
\text { oMTSS) }\end{array}$ & $\begin{array}{l}\text { Desarnolio e } \\
\text { innovación }\end{array}$ & IDICT & OSDE2 & $\begin{array}{l}\text { Calle } 18 \text { A entre } 41 \text { y } \\
47\end{array}$ & 0 & isctggnal com & Eimmploz & Ejemplo3 \\
\hline
\end{tabular}
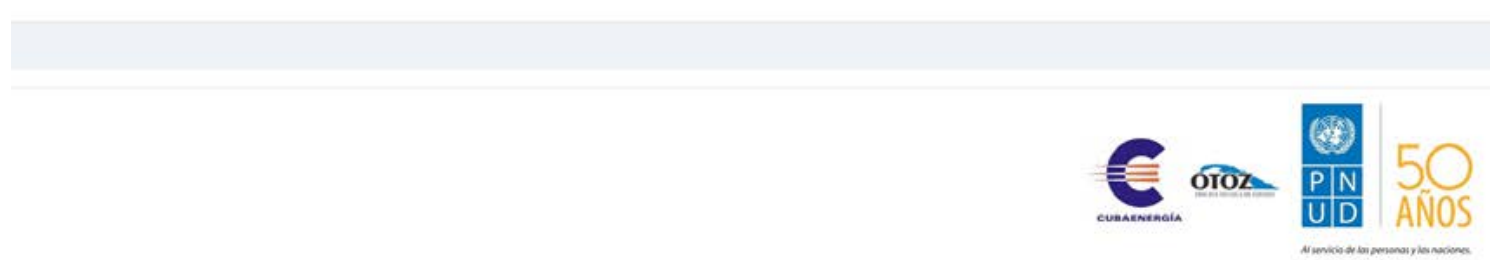

Figura 4. Vista de reportes

Fuente: elaboración propia.

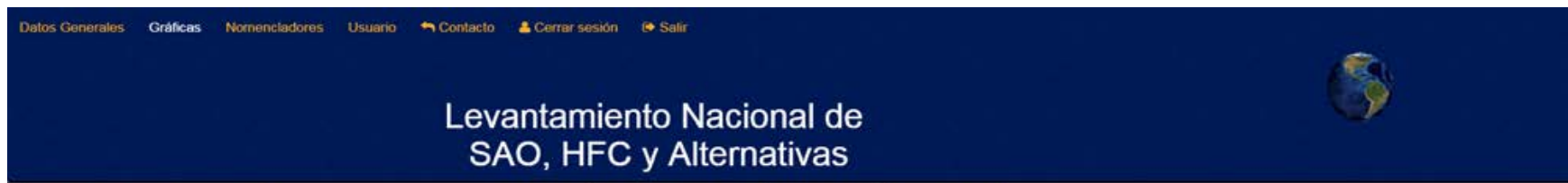

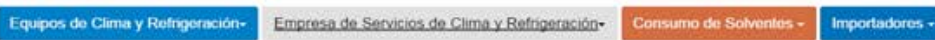
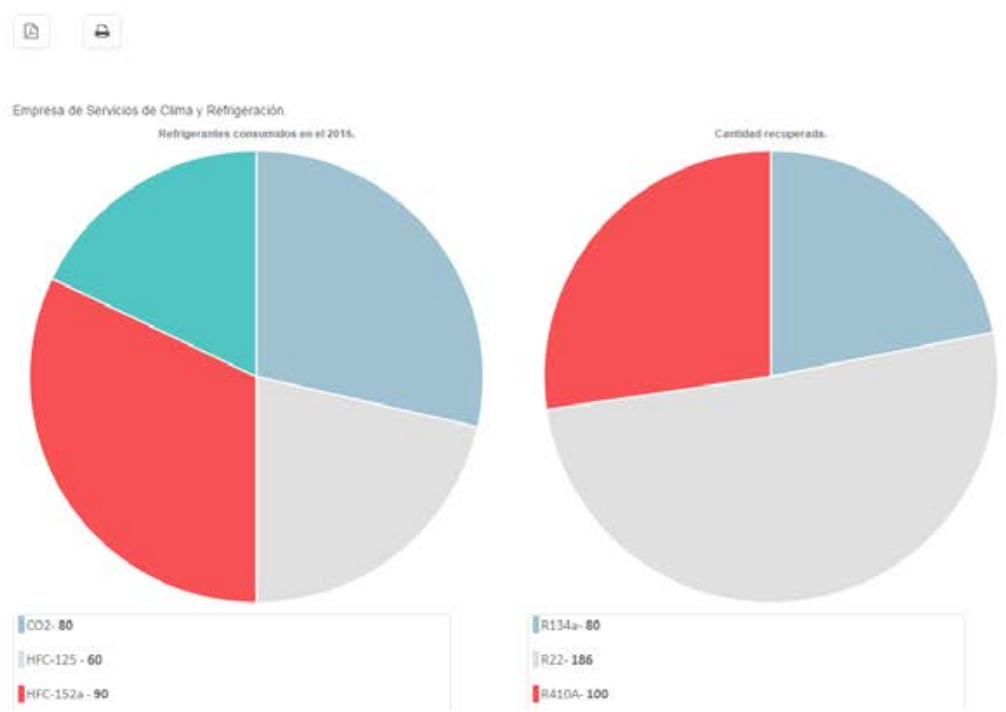

Figura 5. Reporte por gráficas del uso de SAO

Fuente: elaboración propia. 


\section{Trabajos relacionados}

Son notables los sistemas de inventario química que parcialmente recogen detalles y seguimiento de sustancias químicas, clasificándolas de manera general. Chemical Inventory Management and Software (Webb, 2013). Sin embargo, al tener un ámbito de aplicación general conlleva a que ajustar el proceso de control específicamente de las SAO sea complejo, al estar enfocado solo en las sustancias químicas y no en los equipos que la emplean. En el ámbito de las SAO se pueden encontrar herramientas como webODS (Regaction, 2017), específicamente dedicada al control de las SAO; de manera general esta posee características que permiten:

- Control de refrigerantes.

- Mantenimiento y certificación técnica.

- Registro de operaciones.

- Notificaciones de seguimiento.

- Monitoreo de sustancias.

- Reportes.

Sin embargo, a pesar de estar dedicada al sector de las SAO funciona independiente por entidad, por lo que la centralización de los datos se transforma en un proceso externo a su ámbito de aplicación.

\section{Conclusiones}

La gestión y control de las sustancias que afectan la capa de ozono es una tarea que se debe tener en cuenta en cada empresa que contenga equipos que trabajen con ellas. En Cuba, existe la entidad de la Oficina Técnica del Ozono encargada de coordinar y controlar el programa nacional de implementación del Protocolo de Montreal. La utilización de herramientas informáticas permite que este proceso de control se realice de manera eficaz, eficiente y centralizada. Con ello se logra un mayor control de todas las sustancias involucradas. El desarrollo de la herramienta para el levantamiento con tecnologías libres contribuye al desarrollo local del país y a la soberanía tecnológica. Como resultado de la investigación, la aplicación de software permite la gestión de inventario de las sustancias, de equipos, empresas y servicios, así como levantamiento del estado técnico y la centralización de la información para realizar análisis globales.

\section{REFERENCIAS}

Chacon, S. (2014). Pro Git. Recuperado de http://pywechat.qiniudn.com/progit.zh.pdf

Díaz, V.G., Valdez, E.R.N., Espada, J.P., Bustelo, C.P.G., Lovelle, J.M.C. y Marín, C.E.M. (2014). Introducción breve a la ingeniería dirigida por modelos. Tecnura, 18(40), 127-142. http://revistas.udistrital. edu.co/ojs/index.php/tecnura/article/view/6980

GitHub. (2016). Documentation | Electron. Recuperado de https://electron.atom.io/docs/

Gómez, F. S., Ordóñez, A., \& Solarte, M. F. (2015). Marco de referencia para la integración de recursos web como servicios de e-learning en .LRN. Tecnura, 19(46). https://doi.org/10.14483/udistrital.jour. tecnura.2015.4.a06

Freeman, A. (2014). PRO ANGULAR JS. Nueva York: APRESS.

Intergovernmental Panel on Climate Change y Grupo de Evaluación Tecnológica y Económica. (2005). La protección de la capa de ozono y el sistema climático mundial: cuestiones relativas a los hidrofluorocarbonos y a los perfluorocarbonos. Grupo Intergubernamental de Expertos sobre el Cambio Climático. Convención Marco de las Naciones Unidas sobre el Cambio Climático y el Protocolo de Montreal. Recuperado de https://www.ipcc.ch/pdf/ special-reports/sroc/sroc_spmts_sp.pdf

Miller, J., Tischer, D.M., Vosseler, C., Bark, K. y Weick, V. (2004). Guía para la gestión de sustancias químicas Cómo optimizar la gestión de sustancias químicas. Eschborn: Deutsche Gesellschaft fürTechnische Zusammenarbeit (GTZ) GMBH. Recuperado de https://www.coursehero.com/file/26275230/ articles-37251-recurso-1 pdf/ 
Montzka, S., Reimann, S., O’Doherty, S., Engel, A. y Krüger, K. (2011). Ozone-depleting substances (ODSs) and related chemicals. Ozone-depleting substances (ODSs) and related chemicals. Montreal: World Meteorological Organization. Recuperado de http://ws680.nist.gov/publication/get_pdf. cfm?pub_id=909747

Palacios, J. (2012). Metodología SCRUM. Recuperado de https://es.slideshare.net/jeffersonpalacios3/ metodologa-scrum-15093551

Programa de Naciones Unidas para el Medio Ambiente (PNUMA) (2007). Manual del Protocolo de Montreal relativo a las sustancias que agotan la capa de ozono. 7a. ed. Nairobi: Convenio de Viena para la Protección de la Capa de Ozono; Protocolo de Montreal.

Pokorny, J. (2013). NoSQL databases: a step to database scalability in web environment. International Journal of Web Information Systems, 9(1), 69-82. http:// doi.org/10.1108/17440081311316398

Poveda, A.F., González, J.A.M., Velandia, J.B. y Villalobos, O.A.R. (2013). Diseño y desarrollo de un prototipo para la integración y visualización de recursos web semánticos mediante grafos conceptuales. Tecnura, 17, 62-78. http://revistas.udistrital. edu.co/ojs/index.php/tecnura/article/view/7238

Regaction (2017). webODS. Houston: XPRT Sourcing.

Rivas, L., Pérez, M., Mendoza, L. y Grimán, A. (2010). Criterios para la selección de herramientas de ingeniería de software en PYMES. Revista de la Facultad de Ingeniería Universidad Central de Venezuela, 25(1), 89-104.

Romero Rodriguez, F.I. y San Juan, Y. (2017). Prácticas para el desarrollo multiplataforma con base de Electron utilizando AngularjS y PouchDB. Monografías, 1-7.

San Juan, Y. (2017). Levantamiento SAO y HFC. Habana: CITMATEL.

Schwaber, K. y Sutherland, J. (2013). The Scrum Guide. Recuperado de https://www.scrumguides.org/docs/ scrumguide/v1/scrum-guide-us.pdf

Smith, G. y Harvey, D. (2017). PouchDB, the JavaScript Database that Syncs! Recuperado de https://pouchdb.com/

URS. (2009). Management of Ozone Depleting Substances. In Environmental Focus. En Environmental Focus (pp. 1-44). Louisiana: Louisiana Air \& Waste Management Association. Recuperado de https://aawma.org/wp-content/uploads/2017/10/2009_3-5. pdf

Webb, J. (2013). Best Practices for Managing Laboratory Chemical Inventory. Catalyst, 5. Recuperado de https://media.vwr.com/emdocs/docs/Best_Practices_for_Managing_Laboratory_Chemical_Inventory.pdf

Xalambrí, S.D. (2016). Desarrollando aplicaciones de escritorio con Electron.js. Recuperado de https:// platzi.com/blog/aplicaciones-escritorio-electron-js/

\section{(C) $(1) \Theta$}

Tecnura • p-ISSN: 0123-921X • e-ISSN: 2248-7638 • Vol. 21No. $54 \bullet$ Octubre - Diciembre de $2017 \bullet$ pp. 59-67 